\title{
ON QUADRATIC DIFFERENTIALS WITH CLOSED TRAJECTORIES ON OPEN RIEMANN SURFACES
}

\author{
KURT STREBEL
}

\section{Introduction}

A holomorphic or meromorphic quadratic differential $\varphi$ on a Riemann surface $R$ is represented by a system of holomorphic resp. meromorphic function elements $\varphi_{\nu}\left(z_{v}\right)$ in the local parameters $z_{v}$ such that the expression $\varphi_{v}\left(z_{v}\right) d z_{v}^{2}$ stays invariant under a conformal transformation of the parameter. In the sequel we will always leave the index $v$ away and just write $\varphi(z) d z^{2}$. The horizontal trajectories of $\varphi$ are the maximal curves $\alpha$ on $R$ along which $\varphi(z) d z^{2}$ is real and positive. Thus zeroes and poles are excluded by definition on a trajectory; but of course it can tend to such a point in either direction. In this case the trajectory is called critical, otherwise regular.

The closed trajectories of a quadratic differential $\varphi$ (if there are any) sweep out certain disjoint ringdomains $R_{i}$ of $R$ which we call the characteristic ringdomains of $\varphi$. On compact surfaces there are holomorphic quadratic differentials with the property that all regular horizontal trajectories are closed. The induced geometric structure on $R$ can now be used to characterize the quadratic differentials with closed trajectories (see first part of Section 3).

It is the purpose of this paper to generalize these structure theorems to open (i.e. non compact) Riemann surfaces. But now $\varphi$ can have infinitely many characteristic ringdomains. It is then said to be of infinite, otherwise of finite (topological) type. It is this last case we are going to deal with. One can roughly say that everything what is true on compact surfaces is also true, in the finite case, on arbitrary open surfaces.

This paper is closely related to the papers [1], [2], [3] of the author. However, we only make use of the main existence theorem on compact Riemann surfaces, which is proved in [1]. This theorem is then first generalized, by the process of doubling, to compact bordered surfaces. The rest 
is done by exhaustion. The case of compact surfaces with punctures is contained in the general result to be proved, but now no branched covering surfaces are needed. Also the quadratic differentials with second order poles, where one has to work with reduced moduli, are treated in the same way. For later generalizations, the extremal property of quadratic differentials with closed trajectories (and finite norm) is proved for arbitrary type.

\section{Extremum properties of quadratic differentials with closed trajectories}

Definition 1. A meromorphic quadratic differential $\varphi$ on an arbitrary Riemann surface $R$ is said to have closed trajectories, if its non closed trajectories cover a set of measure zero. (A point set is said to have measure zero, if its intersection with every parameter neighbourhood has area measure zero, in the respective parameter plane.)

A quadratic differential with closed trajectories cannot have poles of higher order than two, and at every pole of order two the leading coefficient must be negative.

The characteristic ringdomains $R_{i}$ of a quadratic differential are the ringdomains swept out by its closed trajectories. If $\varphi$ has closed trajectories, its characteristic ringdomains fill out the surface up to a set of measure zero.

D efinition 2. A system of finitely or infinitely many Jordan curves $\gamma_{i}$ on a Riemann surface $R$ is called admissible, if none of the curves is homotopically trivial (homotope zero) and if, for $i \neq k, \gamma_{i} \cap \gamma_{k}^{\prime}$ $=\varnothing$ and $\gamma_{i} \sim \gamma_{k}$, where the symbol $\sim$ means free homotopy.

If $\varphi$ is holomorphic on $R$ and if we pick a closed trajectory $\alpha_{i}$ from every characteristic ringdomain $R_{i}$ of $\varphi$, we have an admissible curve system $\left\{\alpha_{i}\right\}$. The same is true for a meromorphic $\varphi$, if we puncture $R$ at the poles of $\varphi$.

Definition 3. A ringdomain $R_{0}$ on $R$ is said to be of homotopy type $\gamma$, if a Jordan curve $\gamma_{0} \subset R_{0}$ which separates its two boundary components is freely homotopic to $\gamma$.

A system of non overlapping ringdomains $R_{j} \subset R$ is said to be of homotopy type $\left\{\gamma_{i}\right\}$, where $\left\{\gamma_{i}\right\}$ is an admissible curve system, if every $R_{j}$ is of homotopy type $\gamma_{i}$ for exactly one $\gamma_{i}$. It is, however, not required that to every $\gamma_{i}$ there really exist a ringdomain $R_{i}$ of this type. (If there is no ringdomain, we sometimes say that it is degenerate and has modulus zero, which allows us to take the same index for corresponding elements of both sets.)

Finally, a holomorphic quadratic differential $\varphi$ with closed trajectories 
is said to be of homotopy type $\left\{\gamma_{i}\right\}$, if its characteristic ringdomains are of this type.

We are now going to prove the basic extremal property of quadratic differentials with closed trajectories and finite norm. For its formulation we need a few notations. We will consider systems of non overlapping ringdomains $\tilde{R}_{j}$ such that the system $\left\{\tilde{\gamma}_{j}\right\}$, where $\tilde{\gamma}_{j}$ is a Jordan curve in $\tilde{R}_{j}$ which separates its boundary components, is admissible. We denote by $a_{j}$ the infimum of the lengths (in the metric $|\varphi(z)|^{1 / 2}|d z|$ ) of all closed curves on $R$ which are freely homotopic to $\tilde{\gamma}_{j}$. For the characteristic ringdomains $R_{i}$ we have $a_{i}=\int_{\alpha_{i}}|\varphi(z)||d z|$ with $\alpha_{i}$ any closed horizontal trajectory in $R_{i} \cdot \tilde{M}_{j}$ and $M_{i}$ are the moduli of $\tilde{R}_{j}$ and $R_{i}$ respectively. The following inequalities hold: $0 \leq a_{j}<\infty, 0<\tilde{M}_{j} \leq \infty$, $0<M_{i}<\infty$.

The orem 1. Let $\varphi$ be a holomorphic quadratic differential with closed trajectories and finite norm

$$
\|\varphi\|=\iint_{R}|\varphi(z)| d x d y
$$

on a Riemann surface $R$. Assume that the curves $\tilde{\gamma}_{j}$ are freely homotopic to (some of) the curves $\alpha_{i}$ or vice versa. Then

$$
\sum_{j} a_{j}^{2} \tilde{M}_{j} \leq\|\varphi\|=\sum a_{i}^{2} M_{i}
$$

where the sum on the left hand side goes over all values $j$ with $a_{j}>0$. Equality holds if and only if the two systems of ringdomains are identical.

Proof. Let $\tilde{R}_{j}$ be a ringdomain with $a_{j}>0$. We map it conformally, first on an annulus, then, after cutting it along a radius, onto a horizontal rectangle in the $z$-plane (the concentric circles going into horizontal straight segments) of base $a_{i}$ and height $\tilde{b}_{i}(\leq \infty)$.

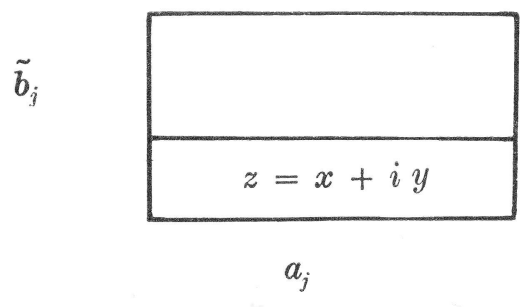

A horizontal interval corresponds to a closed curve which is homotopic to $\tilde{\gamma}_{j}$ and therefore has $\varphi$-length $\geq a_{j}$. If we denote the representation of $\varphi$ in terms of the parameter $z$ by $\varphi(z)$, we have 


$$
a_{j} \leq \int|\varphi(x+i y)|^{1 / 2} d x .
$$

Integration with respect to $y$ and subsequent application of the Schwarz inequality yield

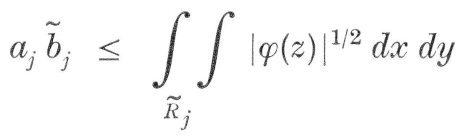

and

$$
a_{j} \tilde{b}_{j} \leq \int_{\widetilde{R}_{j}} \int_{\tilde{R}_{j}}|\varphi(z)| d x d y,
$$

hereby showing that $\tilde{b}_{j}<\infty$.

Summing over the indices $j$, with $a_{j} \tilde{b}_{j}=a_{j}^{2}\left(\tilde{b}_{j} / a_{j}\right)=a_{j}^{2} \tilde{M}_{j}$ we get

$$
\begin{aligned}
\sum_{j} a_{j}^{2} \tilde{M}_{j} & \leq \sum_{j} \int_{\widetilde{R}_{j}} \int|\varphi(z)| d x d y=\int_{\tilde{U}_{j}} \int_{\widetilde{R}_{j}}|\varphi(z)| d x d y \\
& \leq\|\varphi\|_{R}=\sum_{i} a_{i}^{2} M_{i},
\end{aligned}
$$

which establishes the inequality (1). We have not made use, so far, of the homotopy assumptions.

Assume now that equality holds. In the first case, every $\tilde{\gamma}_{j}$ is freely homotopic to a certain closed trajectory $\alpha_{i}$, and we can use the same index. We must have equality in (2) for almost all, hence for all $y$. Therefore the horizontals are going into closed trajectories of $R_{i}$, which means that $\tilde{R}_{i}$ is a subring of $R_{i}$ swept out by closed trajectories. If $\tilde{R}_{i}$ were not identical with $R_{i}$, an open subring of positive $\varphi$-area would be missing, which is impossible. The same argument shows that to every $R_{i}$ there must be an $\tilde{R}_{i}$.

In the second case, for every $\alpha_{i}$ there is a $\tilde{\gamma}_{i}$ and by the same argument, $\tilde{R}_{i}$ is a subring of $R_{i}$ swept out by horizontal trajectories. Let $\tilde{R}_{j}$ be a remaining ringdomain, which does not correspond to an $\alpha_{i}$. The horizontals of the rectangle in the $z$-plane which corresponds to $\tilde{R}_{j}$ must go over into closed geodesics on the surface, in order to have equality in (2). Moreover, $\tilde{R}_{j}$ must have points in common with some $R_{i}$. But if the closed geodesics in $\tilde{R}_{j}$ would not be horizontal, it would intersect $\tilde{R}_{i}$, which is impossible. On the other hand they cannot be horizontal, otherwise $\tilde{\gamma}_{j} \sim \tilde{\gamma}_{i}$ contrary to assumption. Hence there cannot be any $\tilde{R}_{j}$ left over. But then $\tilde{M}_{i}$ must be equal to $M_{i}$ because of (5), hence $\tilde{R}_{i}=R_{i}$ for all $i$. 
The next extremal property is a consequence of theorem one, but not equivalent to it. It has, however, the remarkable feature that it can be formulated without making use of the $\varphi$-metric.

Th e or e m 2. Let $\varphi$ and $\left\{\tilde{R}_{j}\right\}$ be as before. If there are curves $\alpha_{i}$ to which there is no corresponding $\tilde{R}_{i}$, we say that this ringdomain is degenerate and has modulus $\tilde{M}_{i}=0$, and similarly for the curves $\tilde{\gamma}_{j}$. We then use the same index for both sets. With this convention we have

$$
\inf \left\{\frac{\tilde{M}_{i}}{M_{i}}\right\} \leq 1,
$$

and equality holds if and only if $\tilde{R}_{i}=R_{i}$ for all $i$.

Proof. Assume first that the set of free homotopy classes of the curves $\alpha_{i}$ contains the corresponding set for the curves $\tilde{\gamma}_{j}$. We then have because of Theorem 1

$$
\sum_{i} a_{i}^{2} \tilde{M}_{i} \leq \sum_{i} a_{i}^{2} M_{i}
$$

and hence

$$
\sum a_{i}^{2}\left(\tilde{M}_{i}-M_{i}\right) \leq 0
$$

with $a_{i}>0$ for all $i$. Hence $\inf \left\{\tilde{M}_{i}-M_{i}\right\} \leq 0$, and since $M_{i}>0$ for every $i, \inf \left\{\tilde{M}_{i} / M_{i}\right\} \leq 1$. Let equality hold. Then $\tilde{M}_{i} \geq M_{i}$. Thus equality must hold in (7) and we conclude from Theorem 1 that $\tilde{R}_{i}=R_{i}$ for all $i$.

Let now the set of homotopy classes of the curves $\alpha_{i}$ be a subset of the set of homotopy classes of the curves $\tilde{\gamma}_{j}$. We can now write (1) in the form

$$
\sum_{i} a_{i}^{2} \tilde{M}_{i}+\sum_{h} a_{h}^{2} \tilde{M}_{h} \leq \sum_{i} a_{i}^{2} M_{i}
$$

where the sum $\sum_{h}$ goes over the additional ringdomains $\tilde{R}_{j}$. We conclude that

$$
\sum_{i} a_{i}^{2}\left(\tilde{M}_{i}-M_{i}\right) \leq 0
$$

hence $\inf \left\{\tilde{M}_{i} / M_{i}\right\} \leq 1$ and a fortiori $\inf \left\{\tilde{M}_{j} / M_{j}\right\} \leq 1$. Let equality hold. Then

$$
\sum_{i} a_{i}^{2} \tilde{M}_{i}=\sum_{i} a_{i}^{2} M_{i}
$$

and by Theorem 1 again $\tilde{R}_{i}=R_{i}$ for all $i$; in particular, there cannot be any additional ringdomains $\tilde{R}_{j}$. 


\section{Existence proof for compact, bordered surfaces}

The rest of the paper is based on the following existence and uniqueness theorem (see [1]):

Let $\left\{\gamma_{i}\right\}$ be an admissible curve system on a compact surface $R$. Let $\left\{m_{i}\right\}$ be a system of positive numbers. Then there exists a holomorphic quadratic differential $\varphi$ with closed trajectories such that its characteristic ringdomains $R_{i}$ are of homotopy type $\left\{\gamma_{i}\right\}$ and have moduli $M_{i}=\lambda \cdot m_{i}$, for some $\lambda>0$. The differential $\varphi$ is determined up to a positive factor. It is called the solution of the modulus problem for the curves $\gamma_{i}$ with weights $m_{i}$.

Let now $R$ be a compact, bordered surface, with boundary curves $\Gamma_{j}, j=1, \ldots, q$. Let $\left\{\gamma_{i}\right\}_{i=1, \ldots, p}$ denote an admissible curve system on $R$, with weights $m_{i}>0$. The mirror image of $R$ is denoted by $R^{*}$; $\hat{R}=R \cup R^{*}$ is the double, with corresponding boundary points identified. The symmetric image of $\gamma_{i}$ is denoted by $\gamma_{i}^{*}$. Whenever $\gamma_{i}$ is homotopic to some $\Gamma_{j}$, we have $\gamma_{i}^{*} \sim \Gamma_{j}^{*}=\Gamma_{j}$. We therefore disregard $\gamma_{i}^{*}$ in this case (and may of course replace $\gamma_{i}$ by $\Gamma_{j}$ ).

The remaining curve system $\gamma_{i}, \gamma_{i}^{*}$ is admissible on $\hat{R}$. We have to show that $\gamma_{i} \sim \gamma_{k}$ for $i \neq k$, and that $\gamma_{i} \sim \gamma_{k}^{*}$ for all $i$ and $k$ (if there is a $\gamma_{k}^{*}$ ).

Let $\gamma_{i} \sim \gamma_{k}, i \neq k$. Let $D$ be a ringdomain bounded by the two curves. $D$ must have points in common with $R^{*}$, otherwise $\gamma_{i}$ and $\gamma_{k}$ would be homotopic on $R$. But as the boundary $\gamma_{i} \cup \gamma_{k}$ of $D$ is in $R$, $D$ contains all of $R^{*}$. $R^{*}$ must be a subannulus of $D$, bounded by two curves $\Gamma_{1}, \Gamma_{2}$. Then $R$ is an annulus (the symmetric image of $R^{*}$ ), hence $\gamma_{i} \sim \gamma_{k}$ on $R$, a contradiction.

Let $\gamma_{i} \sim \gamma_{k}^{*}$. Again, let $D$ be the ringdomain bounded by $\gamma_{i}$ and $\gamma_{k}^{*}$. $D$ contains points of $R$ as well as of $R^{*}$, hence a boundary curve $\Gamma_{j}$. This curve cannot be homotopic to zero and must therefore be homotopic to both boundary curves of $D$. We have $\gamma_{i} \sim \Gamma_{j}$. But then $\gamma_{i}^{*}$ was left out, hence $i \neq k$. As $\gamma_{i} \sim \gamma_{i}^{*}, \gamma_{i}^{*} \sim \gamma_{k}^{*}$, we conclude $\gamma_{i} \sim \gamma_{k}$, an impossibility.

We now assign weights to the curves of our system: $\gamma_{i} \rightarrow m_{i}$ as before, unless $\gamma_{i} \sim \Gamma_{j}$, in which case we set $\gamma_{i} \rightarrow 2 m_{i}$, and $\gamma_{i}^{*} \rightarrow m_{i}$. Let $\varphi$ be the solution of the modulus problem with these weights. Let $R_{i}, R_{i}^{*}$ be the corresponding ringdomains. The symmetry $T$ of $\hat{R}$ takes the system of curves and weights into itself, hence also the system of ringdomains.

Let $R_{i}$ be the ringdomain associated with $\gamma_{i}$. First assume $\gamma_{i} \sim \Gamma_{j}$ for all $j$. We want to show that then $R_{i} \subset R$. Let $R_{i} \cap R^{*} \neq \varnothing$. If $R_{i} \subset R^{*}$, pick a closed trajectory $\alpha_{i} \subset R_{i}$. As it does not meet $\gamma_{i}$, it bounds, together with $\gamma_{i}$, a ringdomain $D$, which, in its turn evidently 
must contain a boundary curve $\Gamma_{j}$. Thus $\gamma_{i} \sim \Gamma_{j}$, in contradiction to the hypothesis. So let $R_{i}$ contain points of $R$ and of $R^{*}$, hence a boundary point $P$. The symmetry $T$ leaves $P$ invariant and takes $R_{i}$ into $R_{i}^{*}$. Therefore $R_{i} \cap R_{i}^{*} \neq \varnothing$, an impossibility. We conclude: For every curve $\gamma_{i}$ which is not homotopic to a boundary curve $\Gamma_{j}$, the corresponding ringdomain $R_{i}$ lies on $R$.

Assume now $\gamma_{i} \sim \Gamma_{j}$. Then $R_{i}$ must be symmetric (invariant under $T$ ). It therefore contains a boundary curve $\Gamma_{j}$ which must be a line of symmetry. The ringdomain $R_{i} \cap R$ has modulus $m_{i}$. We therefore have solved the modulus problem for the original surface $R$ with the given curves $\gamma_{i}$ and weights $m_{i}$.

Let $P \in \Gamma_{j}$. If $P$ is in one of the ringdomains on $\hat{R}$, it lies on a trajectory (line of symmetry of some $R_{i}$ ). Otherwise it lies on the boundary of one of the ringdomains, hence on a trajectory or else it is a zero of $\varphi$, the restriction of $\hat{\varphi}$ to $R$.

\section{Existence and uniqueness for arbitrary Riemann surfaces}

We proceed to prove the existence theorem for arbitrary Riemann surfaces with finite admissible curve systems. The proof is valid, in particular, for compact surfaces with finitely many distinguished points. As a corollary we find that every surface, except for the sphere with less than four boundary points, carries holomorphic quadratic differentials with finite norm. Another corollary is that ringdomains with maximal moduli are uniquely determined, if the moduli are finite.

Let $\left\{\gamma_{i}\right\}_{i=1, \ldots, p}$ be an admissible curve system on an arbitrary Riemann surface $R$ and let $m_{i}>0$ be the weights. Moreover, assume that the supremum $M\left(\gamma_{i}\right)$ of the moduli of all the ringdomains on $R$ which are of homotopy type $\gamma_{i}$ is finite for every $i$. We put

$$
M=\max \left\{M\left(\gamma_{i}\right) \mid 1 \leq i \leq p\right\} .
$$

For a subdomain of the sphere this rules out domains with less than four boundary points, for then the boundary components would have to be points, and any non trivial Jordan curve $\gamma$ would bound a punctured disk, hence $M(\gamma)=\infty$. On the other hand, let $R$ be a subdomain of the Riemann sphere with at least four boundary points. Then either the boundary contains a continuum or we have a non trivial curve $\gamma$ which does not bound a puctured disk. For surfaces of positive genus we can pick a curve which does not disect the surface. Then its maximal modulus is finite, as it would otherwise be the boundary of a puctured disk on $R$, hence clearly disect the surface. This proves the first corollary. 
To prove uniqueness, let $\varphi$ and $\tilde{\varphi}$ be two solutions, with ringdomains $R_{i}$ and $\tilde{R}_{i}$ of homotopy type $\left\{\gamma_{i}\right\}$, and moduli $M_{i}$ and $\tilde{M}_{i}$ respectively. Then the extremal property of Theorem 2, applied to both $\varphi$ and $\tilde{\varphi}$, immediately shows that $R_{i}=\tilde{R}_{i}$ for all $i$. As the trajectories are the same in $R_{i}$, the quotient $\tilde{\varphi} / \varphi$ must be real and positive, hence a positive constant. Because of the normalization $\|\varphi\|=\|\tilde{\varphi}\|=1$, we must have $\tilde{\varphi}=\varphi$.

The existence of a solution is shown by a limiting process. Consider an exhaustion of $R$ by compact bordered surfaces $R^{(n)}, n=1,2, \ldots$. We can assume, without loss of generality, that the curves $\gamma_{i}$ are in $R^{(n)}$ for all $n$. We can therefore solve the modulus problem for the curve system $\left\{\gamma_{i}\right\}$ and the weights $m_{i}$ on $R^{(n)}$. Let $\varphi_{n}$, with norm $\left\|\varphi_{n}\right\|=1$, be the solution. The ringdomains, moduli etc. are denoted by $R_{\text {in }}, M_{\text {in }}=$ $b_{\text {in }} / a_{\text {in }}$ etc. I claim that the sequence $\left(\varphi_{n}\right)$ tends in norm (hence locally uniformly) towards a quadratic differential $\phi$ on $R$ with closed trajectories, the characteristic ringdomains $R_{i}$ of which solve the modulus problem with curves $\gamma_{i}$ and weights $m_{i}$ for $R$.

Proof. As the norm of the $\varphi_{n}$ is one, we can clearly select, by a diagonal process, a locally uniformly convergent subsequence, which we again denote by $\left(\varphi_{n}\right)$. Its limit $\varphi$ is a holomorphic quadratic differential on $R$ with norm $\|\varphi\| \leq 1$. We have $M_{i n}=\lambda_{n} m_{i}$. As the system of ringdomains $R_{\text {in }}$ satisfies the homotopy conditions on $R^{(n+1)}$,

$$
\operatorname{Min}\left\{M_{i n}\left|M_{i(n+1)}\right| 1 \leq i \leq p\right\} \leq 1 \text {, }
$$

hence $\lambda_{n} \leq \lambda_{n+1}$. On the other hand, $M_{i n}=\lambda_{n} \cdot m_{i} \leq M$ for all $i$ and $n$, hence $\lambda_{n} \leq M / m$, with $m=\operatorname{Max}\left\{m_{i} \mid 1 \leq i \leq p\right\}$. We conclude that the coefficients $\lambda_{n}$ converge to their supremum $\lambda$, moreover $M_{\text {in }}$ $\rightarrow M_{i}=\lambda m_{i}$ for all $i$. This is of course true for the original sequence.

For fixed $i$ the function $g_{i n}=\exp \left(2 \pi i / a_{i n}\right) \Phi_{n}(P)$ with $\Phi_{n}=$ $\int\left(\varphi_{n}(z)\right)^{1 / 2} d z$ is a $1-1$ conformal mapping of $R_{\text {in }}$ onto a circular annulus in the z-plane. By choosing the sign of $\Phi_{n}$ and the integration constant properly, we can assume that the orientation induced by $\gamma_{i}$ (the Jordan curves $\gamma_{i}$ are supposed to be oriented) is taken into the positive orientation of the $z$-plane and the "outer" boundary component of $R_{\text {in }}$ goes into $|z|=1$. (This only determines the imaginary part of the integration constant, the real part still being free. The mapping $g_{\text {in }}$ is determined up to a rotation.) The inverse $f_{i n}=g_{\text {in }}^{-1}$ is a $1-1$ conformal mapping of $r_{\text {in }}<$ $|z|<1, M_{\text {in }}=(1 / 2 \pi) \log \left(1 / r_{\text {in }}\right)$, onto $R_{\text {in }}$.

By passing, if necessary, to a subsequence, we may assume that the mappings $f_{\text {in }}, n \rightarrow \infty$, tend locally uniformly to a $1-1$ conformal mapping $f_{i}: r_{i}<|z|<1 \rightarrow R_{i}$. 


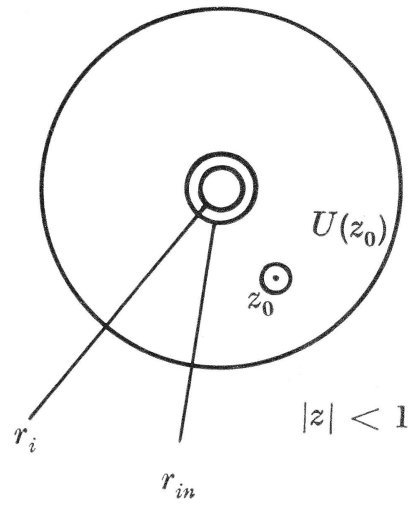

Consider a neighbourhood of a point $z_{0}, \quad r_{i}<\left|z_{0}\right|<1$. We may introduce $z$ as a parameter in this neighbourhood (in fact in all of $R_{i}$ ). The mapping $f_{i}$ becomes, in terms of this parameter, the identity. Moreover $g_{\text {in }}(z) \rightarrow z$ uniformly in $U\left(z_{0}\right)$. Let $z_{n}=g_{\text {in }}(z)$. Then the following equations hold:

$$
\begin{aligned}
z_{n} & =\exp \frac{2 \pi i}{a_{i n}} \Phi_{n}(z), \\
\log z_{n} & =\frac{2 \pi i}{a_{i n}} \Phi_{n}(z), \\
\frac{1}{z_{n}} \frac{d z_{n}}{d z} & =\frac{2 \pi i}{a_{i n}} \Phi_{n}^{\prime}(z), \\
\left(\frac{z_{n}^{\prime}}{z_{n}}\right)^{2} & =-\left(\frac{2 \pi}{a_{i n}}\right)^{2} \varphi_{n}(z) .
\end{aligned}
$$

As, for $n \rightarrow \infty, z_{n} \rightarrow z, z_{n}^{\prime} \rightarrow 1, a_{i n} \rightarrow a_{i}$ (taking a subsequence again, if necessary), we have

$$
\varphi(z)=-\left(\frac{a_{i}}{2 \pi}\right)^{2} \frac{1}{z^{2}} .
$$

Thus, for $d z=i \cdot z$, we have $\varphi(z) d z^{2}=\left(a_{i} / 2 \pi\right)^{2} d z^{2}>0$. The circles $|z|=$ const are closed trajectories of $\varphi$. Moreover

$$
\begin{aligned}
1 \geq\|\varphi\| & \geq \sum_{i} \int_{R_{i}} \int_{n}|\varphi(z)| d x d y=\sum_{i} a_{i}^{2} M_{\imath} \\
& =\lim _{n \rightarrow \infty} \sum_{i} a_{i n} M_{i n}=1 .
\end{aligned}
$$

The set $R \backslash \cup R_{i}$ has measure zero. Hence $\varphi$ has closed trajectories and the $R_{i}$ are the ringdomains of $\varphi$. They obviously belong to the curve system $\left\{\gamma_{i}\right\}$. 
The sequence $\left(\varphi_{n}\right)$ is locally bounded (i.e. $\varphi_{n}(z)$ in terms of a fixed local parameter $z$ is a locally bounded sequence of holomorphic function elements). Therefore, by the Cauchy representation formula, the same is true for the derivatives $\varphi_{n}^{\prime}(z)$. The sequence is therefore equicontinuous, and any convergent subsequence converges locally uniformly. If the original sequence $\left(\varphi_{n}\right)$ would not converge towards $\varphi$ at some point $P$, there would be a subsequence with a $\operatorname{limit}_{n \rightarrow \infty} \varphi_{n}(z) \neq \varphi(z)$. Starting out with this sequence we would arrive at some quadratic differential $\tilde{\varphi} \neq \varphi$ which is a solution of the same modulus problem, contradicting the uniqueness of the solution.

\section{The ringdomains of a given homotopy type}

Let $\left\{\gamma_{i}\right\}_{i=1, \ldots, p}$ be a finite admissible curve system, with finite maximal moduli $M\left(\gamma_{i}\right)$ (= supremum of the moduli of all ringdomains of homotopy type $\gamma_{i}$ on $R$ ), on an arbitrary Riemann surface $R$. We are looking at the $p$-tuples of moduli of all $p$-tuples of non overlapping ringdomains of homotopy type $\left\{\gamma_{i}\right\}$. It is obviously enough to consider, in each direction $\left\{m_{i}\right\}$, the extremal system. To achieve compactness we now allow some of the $m_{i}$ to be zero.

Let $\vec{m}=\left(m_{1}, m_{2}, \ldots, m_{p}\right)$ be an arbitrary unitvector with non negative coordinates $m_{i} \geq 0, i=1, \ldots, p$. Let $\left\{R_{i}\right\}$ be the extremal system of ringdomains of homotopy type $\left\{\gamma_{i}\right\}$ in the direction $\vec{m}$. This means that $\left\{R_{i}\right\}$ is the system of characteristic ringdomains of a holomorphic quadratic differential $\varphi$ with closed trajectories and that $M_{i}=\lambda m_{i}$ for all $i$, which we abbreviate to $\vec{M}=\lambda \vec{m}$. Of course, $M_{i}=0$ means that there is no ringdomain (no closed trajectory) of homotopy type $\gamma_{i}$.

Theorem 3. The moduli vector $\vec{M}=\left(M_{1}, M_{2}, \ldots, M_{p}\right)$ depends continuously on its direction $\vec{m}$.

Proof. The proof is given in two steps. First we consider an arbitrary sequence of normalized holomorphic quadratic differentials $\varphi_{n}\left(\left\|\varphi_{n}\right\|=1\right)$, with closed trajectories and of homotopy type $\left\{\gamma_{i}\right\}$. We can pick a locally uniformly convergent subsequence, which we again denote by $\left(\varphi_{n}\right)$. Let $\varphi=\lim _{n \rightarrow \infty} \varphi_{n}$. We claim that $\left\|\varphi_{n}-\varphi\right\| \rightarrow 0$, that $\varphi$ has closed trajectories, that it is of type $\left\{\gamma_{i}\right\}$, that $M_{\text {in }} \rightarrow M_{i}$ for all $i=1, \ldots, p$, and finally that $a_{i n} \rightarrow a_{i}$ for all $i$. (The latter follows in the course of the proof for those indices $i$, for which $M_{i}>0$; it will later be shown that it is true for all $i$.)

As $M_{i n} \leq M\left(\gamma_{i}\right), i=1, \ldots, p, n=1,2, \ldots$ we can pick a subsequence for which the sequences of moduli $M_{\text {in }}$ converge for all $i$. We denote all subsequences by $\left(\varphi_{n}\right)$ and will later show that it is in fact true for the 
original sequence. Let $M_{i}=\lim _{n \rightarrow \infty} M_{i n}$. We put $i=k$ for $M_{i}=0$, $i=h$ for $M_{i}>0$. For all $h$ we set

$$
g_{h n}(P)=\exp \frac{2 \pi i}{a_{h n}} \Phi_{n}(P),
$$

the imaginary constant of $\Phi_{n}(P)$ in $R_{h n}$ being chosen in such a way that the outer radius of the image annulus is equal to one, the inner radius is equal to $r_{h n}$, with $M_{h n}=(1 / 2 \pi) \log \left(1 / r_{h n}\right)$. Let $f_{h n}=g_{h n}^{-1}$. For a properly chosen subsequence the mappings $f_{h n}$ converge locally uniformly to some $1-1$ conformal mappings $f_{h}: r_{h}<|z|<1 \rightarrow R_{h}, r_{h}=\lim _{n \rightarrow \infty} r_{h n}$, of the limit annuli onto disjoint ringdomains $R_{h} . R_{h}$ is evidently of homotopy type $\gamma_{h}$.

By means of the mapping $f_{h}$ we can introduce the parameter $z$ in $R_{h}$. Then, $\left(g_{h n}\right)$ becomes a sequence of mappings which tends locally uniformly in $r_{h}<|z|<1$ towards the identity. We have

$$
\begin{gathered}
g_{h n}(z)=\exp \frac{2 \pi i}{a_{h n}} \Phi_{n}(z), \\
\log g_{h n}(z)=\frac{2 \pi i}{a_{h n}} \Phi_{n}(z)
\end{gathered}
$$

and for the derivative with respect to $z$

$$
\frac{g_{h n}^{\prime}(z)}{g_{h n}(z)}=\frac{2 \pi i}{a_{h n}} \Phi_{n}^{\prime}(z) .
$$

Taking squares and going to the limit we get, as $g_{h n}^{\prime}(z) \rightarrow 1$,

$$
\frac{1}{z^{2}}=-\left(\frac{2 \pi}{a_{h}}\right)^{2} \varphi(z) .
$$

(Here it follows that $a_{h}=\lim _{n \rightarrow \infty} a_{h n}$ exists.)

From this we easily recognize that the circles $|z|=$ const are closed trajectories of $\varphi$. Hence $R_{h}$ is a subring of a characteristic ringdomain of $\varphi$, swept out by closed trajectories. The $p$-length of these trajectories is obviously $a_{h}$, and the $\varphi$ area of $R_{h}$ is $a_{h}^{2} M_{h}$. Since $\varphi$ is the locally uniform limit of the $\varphi_{n},\left\|\varphi_{n}\right\|=1$, we have $\|\varphi\| \leq 1$. We get

$$
1 \geq\|\varphi\| \geq \sum_{h} a_{h}^{2} M_{h}=\lim _{n \rightarrow \infty} \sum a_{h n}^{2} M_{h n} \leq \lim _{n \rightarrow \infty} \sum a_{i n}^{2} M_{i n}=1 .
$$

Therefore $\|\varphi\|=\sum a_{h}^{2} M_{h}=1$. The ringdomains $R_{h}$ cover $R$ up to a set of measure zero. Therefore $p$ has closed trajectories and is of type $\left\{\gamma_{i}\right\}$. Obviously $M_{i n} \rightarrow M_{i}$ for all $i=1, \ldots, p$, and $a_{h n} \rightarrow a_{h}\left(\right.$ for $\left.M_{h}>0\right)$. 
An easy argument shows that $\left\|\varphi_{n}-\varphi\right\| \rightarrow 0$, as $\varphi_{n} \rightarrow \varphi$ locally uniformly and $\left\|\varphi_{n}\right\|=\|\varphi\|=1$.

We started with a sequence $\left(\varphi_{n}\right)$ which converges locally uniformly towards $\varphi$. Then there exists a subsequence $\left(\varphi_{n_{v}}\right)$ with $\left\|\varphi_{n_{v}}-\varphi\right\| \rightarrow 0$. If this were not true for the original sequence we could find a subsequence with a limit $\tilde{\varphi} \neq \varphi$. But a subsequence of this would again tend to $\varphi$, a contradiction. Therefore $\left\|\varphi_{n}-\varphi\right\| \rightarrow 0$. Moreover, as $\varphi$ determines its characteristic ringdomains, $M_{i n} \rightarrow M_{i}, a_{h n} \rightarrow a_{h}$ for all $i$ resp. for all $h$ with positive $M_{h}$.

The second step of the continuity proof is short. Let $\vec{m}_{n} \rightarrow \vec{m}$. Assume $\vec{M}_{n} t \rightarrow \vec{M}$. Then we can pick a convergent subsequence, which we denote again by $\left(\vec{M}_{n}\right)$, such that $\vec{M}_{n} \rightarrow \lambda_{0} \vec{m}=\vec{M}_{0} \neq \vec{M}$. Let $\varphi_{n}$ be the quadratic differential, associated with the system $\left\{\gamma_{i}\right\}$ and weight $\vec{m}_{n}$. A subsequence of this sequence converges towards a quadratic differential $\varphi_{0}$ of homotopy type $\left\{\gamma_{i}\right\}$ with moduli vector $\vec{M}_{0}$. But $\vec{M}$ is supposed to be the moduli vector in the direction $\vec{m}$, and because of the uniqueness $\vec{M}_{0}=\vec{M}$, a contradiction. The continuity is proved.

The surface which is described by $\vec{M}$ as a function of the unit vector $\vec{m}$ is called the surface of moduli associated with $\left\{\gamma_{i}\right\}:$ We denote it by $\mathfrak{M}=\mathfrak{M}\left(\left\{\gamma_{i}\right\}\right)$.

Convexity, tangent plane of $\mathfrak{M}$. Let $\vec{M}_{0}$ be an arbitrary point of $\mathfrak{M}$. Let $\varphi_{0}$ be the holomorphic quadratic differential with the moduli vector $\vec{M}_{0},\left\|\varphi_{0}\right\|=1$. We put $\vec{a}_{0}=\left(a_{01}^{2}, \ldots, a_{0 p}^{2}\right)$, with $a_{0 i}$ the infinum of the lengths of all closed curves which are homotopic to $\gamma_{i}$, in the $\varphi$-metric. Then the following can easily be proved:

(1) The plane $\left(\vec{a}_{0}, \vec{x}-\vec{M}_{0}\right)=0$ has only the point $\vec{M}_{0}$ in common with $\mathfrak{M}$. For let $\vec{M} \in \mathfrak{M}$. The corresponding ringdomains are of homotopy type $\left\{\gamma_{i}\right\}$, hence

$$
\left(\vec{a}_{0}, \vec{M}\right) \leq\left(\vec{a}_{0}, \vec{M}_{0}\right)
$$

with equality only for $\vec{M}=\vec{M}_{0}$. We conclude

$$
\left(\vec{a}_{0}, \vec{M}-\vec{M}_{0}\right)<0
$$

unless $\vec{M}=\vec{M}_{0}$. The entire surface $\mathfrak{M}$, hence also the set bounded by $\mathfrak{M}$ and the coordinate planes, lie to the left (*) of the plane $\left(\vec{a}_{0}, \vec{x}-\vec{M}_{0}\right)=0$.

(2) The plane $\left(\vec{a}_{0}, \vec{x}-\vec{M}_{0}\right)=0$ is the tangent plane. This is easily proved for interior points of $M$ (i.e. $M_{0 i}>0$ for all $i$ ). For at such a point the vector $\vec{a}$ is continuous: $\vec{M}_{n} \rightarrow \vec{M}_{0} \Rightarrow \vec{a}_{n} \rightarrow \vec{a}_{0}$. We have the two inequalities

(*) I.e. the same side as the origin. 


$$
\begin{aligned}
& \left(\vec{a}_{0}, \vec{M}_{n}\right) \leq\left(\vec{a}_{0}, \vec{M}_{0}\right) \\
& \left(\vec{a}_{n}, \vec{M}_{0}\right) \leq\left(\vec{a}_{n}, \vec{M}_{n}\right),
\end{aligned}
$$

hence, with

$$
\begin{gathered}
\vec{e}_{n}=\frac{\vec{M}_{n}-\vec{M}_{0}}{\left|\vec{M}_{n}-\vec{M}_{0}\right|}, \\
\left(\vec{a}_{0}, \vec{e}_{n}\right) \leq 0, \\
\left(\vec{a}_{n}, \vec{e}_{n}\right)=\left(\vec{a}_{0}, \vec{e}_{n}\right)+\left(\vec{a}_{n}-\vec{a}_{0}, \vec{e}_{n}\right) \geq 0 .
\end{gathered}
$$

We conclude: $-\left(\vec{a}_{n}-\vec{a}_{0}, \vec{e}_{n}\right) \leq\left(\vec{a}_{0}, \vec{e}_{n}\right) \leq 0$, consequently $\lim _{n \rightarrow \infty}\left(\vec{a}_{0}, \vec{e}_{n}\right)$ $=0$.

At interior points $\mathfrak{M}$ therefore has a continuously changing tangent plane with normal vector $\vec{a}$.

To prove the same at a boundary point, let $\vec{M}_{0}$ be a boundary vector. Let $M_{h 0}>0, M_{k 0}=0$. We have

$$
\begin{gathered}
-\sum_{h}\left(a_{h n}^{2}-a_{h 0}^{2}\right) \cdot e_{h n}-\sum_{k}\left(a_{k n}^{2}-a_{k 0}^{2}\right) \cdot e_{k n} \\
\leq \sum_{i} a_{i 0}^{2} \cdot e_{i n} \leq 0 .
\end{gathered}
$$

For arbitrary index $i$ we have $\varlimsup_{n \rightarrow \infty} a_{i n} \leq a_{i 0}$ :

This is easily seen, for, let $\gamma_{i}$ be an arbitrary element of its homotopy class. Then

$$
a_{\text {in }} \leq \int_{\gamma_{i}}\left|\varphi_{n}(z)\right|^{1 / 2}|d z| \rightarrow \int_{\gamma_{i}}|\varphi(z)|^{1 / 2}|d z| .
$$

The last expression becomes smaller than $a_{i}+\varepsilon, \varepsilon>0$, for properly chosen $\gamma_{i}$.

Now, for all indices $h, a_{h n} \rightarrow a_{h 0}$. For the indices $k$ we have

$$
e_{k n}=\frac{M_{k n}-M_{k 0}}{\left|\vec{M}_{k n}-\vec{M}_{k 0}\right|},
$$

hence $0<e_{k n} \leq 1$.

For given $\varepsilon>0$ we can find $N$ such that for $n>N$ and all $h$ resp. $k$

$$
\begin{gathered}
\left|a_{h n}^{2}-a_{h 0}^{2}\right|\left|e_{h n}\right|<\varepsilon, \\
\left(a_{k n}^{2}-a_{k 0}^{2}\right)<\varepsilon
\end{gathered}
$$

and therefore also $\left(a_{k n}^{2}-a_{k 0}^{2}\right) e_{k n}<\varepsilon$. The left hand side of the above inequality then becomes $>-p \varepsilon$. We have proved 


$$
\lim _{n \rightarrow \infty}\left(\vec{a}_{0}, \vec{e}_{n}\right)=0
$$

also for all boundary points of $\mathfrak{M}$. This surface therefore has a normal vector at each point, namely $\vec{a}=\left(a_{1}^{2}, a_{2}^{2}, \ldots, a_{p}^{2}\right)$.

It is also immediately seen, from the above inequality, that $\vec{a}_{n} \rightarrow \vec{a}_{0}$ if $\vec{M}_{0}$ is approached in a fixed direction. For then, the unit vector $\vec{e}$ does not depend on $n$. We have

$$
\sum_{h}\left(a_{h n}^{2}-a_{h 0}^{2}\right) e_{h}+\sum_{k}\left(a_{k n}^{2}-a_{k 0}^{2}\right) e_{k} \geq 0 .
$$

Assuming $e_{k}>0, \lim _{n \rightarrow \infty} a_{k n}<a_{k 0}$ would lead to a contradiction.

\section{Quadratic differentials with second order poles. Extremal property}

Let $\varphi$ be a holomorphic quadratic differential with closed trajectories which is of finite topological type on an arbitrary Riemann surface $R$. We assume that $\phi$ has infinite norm. Then at least one of the characteristic ringdomains of $\varphi$ must have infinite modulus. We exclude the twice punctured sphere, which means that every ringdomain $R_{j}$ with infinite modulus can be mapped conformally onto a punctured disk. We can then add the puncture as a point $Q_{j}$ to the surface $R$, and consequently introduce a conformal parameter $z_{j}$ near $Q_{j}$, such that $Q_{j}$ corresponds to $z_{j}=0$. The quadratic differential $\varphi$ has a second order pole at $z_{j}=0$ with a real, negative leading coefficient $-A_{j}$ which is independent of the choice of the parameter. Let $M_{j}$ be the reduced modulus of $R_{j}$ with respect to the parameter $z_{j}$ (i.e. $M_{j}=(1 / 2 \pi) \log r_{j}$, where $r_{j}$ is the mapping radius with respect to $z_{j}$ ). We denote by $R_{k}$ the characteristic ringdomains of $\varphi$ with finite modulus $M_{k}$ and use the index $i$ for the characteristic ringdomains of $\varphi$ without distinction. As before, $a_{i}$ is the length of any closed trajectory $\alpha_{i}$ of $\varphi$ in $R_{i}$ :

$$
a_{i}=\int_{\alpha_{i}}|\varphi(z)|^{1 / 2}|d z| .
$$

Now let $\left\{\tilde{R}_{i}\right\}$ be a system of nonoverlapping ringdomains on $R$ of homotopy type $\left\{\alpha_{i}\right\}$. For every $j, \tilde{R}_{j}$ is to be a punctured disk, with reduced modulus $\tilde{M}_{j}$ taken with respect to the same parameter $z_{j}$. We allow $\tilde{R}_{k}$ to be degenerate (=missing), but not $\tilde{R}_{j}$. Then

$$
\sum_{i} a_{i}^{2} \tilde{M}_{i} \leq \sum_{i} a_{i}^{2} M_{i} \quad(=\text { reduced norm of } \varphi)
$$

with equality holding if and only if $\tilde{R}_{i}=R_{i}$ for all $i$. 
Proof. Let $\zeta_{j}$ be the distinguished parameter for the quadratic differential $\varphi$ near the second order pole $Q_{j}$. The representation of $\varphi$ in terms of this parameter is

$$
\varphi(z) d z^{2}=-\frac{A_{j}}{\zeta_{j}^{2}} d \zeta_{j}^{2}
$$

with $a_{j}=2 \pi A_{j}^{1 / 2}$ the length of the closed trajectories of $R_{j}$. The inequality itself can be shown by cutting out circular holes (in terms of the distinguished parameters) around the points $Q_{j}$ and then applying the inequality for quadratic differentials of finite norm. Letting the radii of the holes tend to zero we arrive at $(10)$. However, in order to discuss the equality sign, we have to be more accurate. So let the domains $\tilde{R}_{j}$ be mapped onto punctured disks $0<\left|\tilde{\zeta}_{j}\right|<\tilde{\varrho}_{j}$ by schlicht functions $\tilde{\zeta}_{j}\left(\zeta_{j}\right)$ with

$$
\frac{d \tilde{\zeta}_{j}}{d \zeta_{j}}\left(Q_{j}\right)=1
$$

The moduli of the ringdomains $\tilde{R}_{j}(\varrho)=\tilde{R}_{j} \backslash\left\{\left|\tilde{\zeta}_{j}\right| \leq \varrho\right\}$ and $R_{j}(\varrho)=$ $R_{j} \backslash\left\{\left|\tilde{\zeta}_{j}\right| \leq \varrho\right\}$ for sufficiently small positive $\varrho$ are denoted by $\tilde{M}_{j}(\varrho)$ and $M(\varrho)$ respectively. Evidently $\tilde{M}_{j}(\varrho)=(1 / 2 \pi) \log \left(\tilde{\varrho}_{j} / \varrho\right)$, and $\tilde{M}_{j}(\varrho)+(1 / 2 \pi) \log \varrho \rightarrow \tilde{M}_{j}, \quad M_{j}(\varrho)+(1 / 2 \pi) \log \varrho \rightarrow M_{j} \quad$ as $\quad \varrho \rightarrow 0$. We cut the annulus $\varrho<\left|\tilde{\zeta}_{j}\right|<\tilde{\varrho}_{j}$ along a radius and map it onto a horizontal rectangle with sides $a_{j}, \tilde{b}_{j}(\varrho)=a_{j} \cdot \tilde{M}_{j}(\varrho)=\left(a_{j} / 2 \pi\right) \log \left(\tilde{\varrho}_{j} / \varrho\right)$ in the $z=x+i y$-plane. As usual, we have

$$
a_{j} \leq \int|\varphi(x+i y)|^{1 / 2} d x
$$

and by the Schwarz inequality

$$
a_{j} \leq \int|\varphi(x+i y)| d x .
$$

Assume that for some $j$ one of the circles $\left|\tilde{\zeta}_{j}\right|=$ const is not a trajectory of $\varphi$. Then there are positive numbers $\varepsilon$ and $\delta$ such that

$$
a_{j}+\varepsilon \leq \int|\varphi(x+i y)| d x
$$

for all $y$ in the $\delta$-neighbourhood of some $y_{0}$. We get by integrating

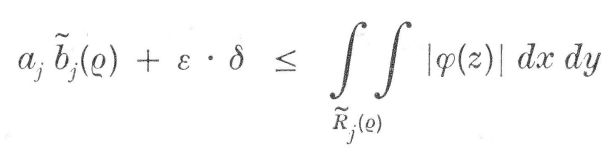

and by summing over the $j$ and $k$ 


$$
\sum_{j} a_{j}^{2} \tilde{M}_{j}(\varrho)+\sum_{k} a_{k}^{2} \tilde{M}_{k}+\varepsilon \delta \leq \sum_{j} a_{j}^{2} M_{j}(\varrho)+\sum_{k} a_{k}^{2} M_{k} .
$$

Adding $\sum_{j} a_{j}^{2} \cdot(1 / 2 \pi) \log \varrho$ to both sides (here we need that there is an $R_{j}$ for every $j$ ) and letting $\varrho \rightarrow 0$ we arrive at a contradiction. (The inequality itself is proved by just dropping the term $\varepsilon \delta$.)

Now we know that the circles $\left|\tilde{\zeta}_{j}\right|=\varrho$ are actually trajectories of $\varphi$. From the equality

$$
\sum_{j} a_{j}^{2} \tilde{M}_{j}+\sum_{k} a_{k}^{2} \tilde{M}_{k}=\sum_{j} a_{j}^{2} M_{j}+\sum_{k} a_{k}^{2} M_{k}
$$

we conclude, subtracting $\sum a_{j}^{2}(1 / 2 \pi) \log \varrho$,

$$
\sum_{j} a_{j}^{2} \tilde{M}_{j}(\varrho)+\sum_{k} a_{k}^{2} \tilde{M}_{k}=\sum_{j} a_{j}^{2} M_{j}(\varrho)+\sum_{k} a_{k}^{2} M_{k}
$$

The right hand side is equal to the norm of $\varphi$ over the truncated surface $R(\varrho)=R \backslash \cup_{j}\left\{\left|\tilde{\zeta}_{j}\right| \leq \varrho\right\}$, because $\varphi$ is a quadratic differential with closed trajectories on $R(\varrho)$ and the $R_{j}(\varrho), R_{k}$ are its characteristic ringdomains. But then by the earlier theorem $\tilde{R}_{j}(\varrho)=R_{j}(\varrho), \tilde{R}_{k}=R_{k}$ for all $j, k$. Hence also $\tilde{R}_{j}=R_{j}$ for all $j$. We have proved

$\mathrm{Th}$ e o r e m 4. Let $\varphi$ be a quadratic differential with closed trajectories and a finite number of characteristic ringdomains on a Riemann surface $R$. We denote by $R_{j}$ the punctured disks (ringdomains with infinite modulus) and by $R_{k}$ the ringdomains with finite modulus. There should be at least one $R_{j}$, and the doubly punctured sphere is of course excluded. Let $\tilde{R}_{j}, \tilde{R}_{k}$ be a system of non overlapping disks and ringdomains, with an $\tilde{R}_{j}$ for every $R_{j}, Q_{j} \in \tilde{R}_{j}$. Then, with the usual notation, but $M_{j}, \tilde{M}_{j}$ the reduced moduli with respect to the same parameters near the second order poles $Q_{j}$, the inequalities

$$
\sum_{j} a_{j}^{2} \tilde{M}_{j}+\sum_{k} a_{k}^{2} \tilde{M}_{k} \leq \sum_{j} a_{j}^{2} M_{j}+\sum_{k} a_{k}^{2} M_{k}
$$

(equality if and only if $\tilde{R}_{j}=R_{j}, \tilde{R}_{k}=R_{k}$ for all $j$ and $k$ ) and, as an easy consequence

$$
\operatorname{Min}_{i=j, k}\left\{\tilde{M}_{i}-M_{i}\right\} \leq 0
$$

(equality if and only if $\tilde{R}_{i}=R_{i}$ for all $i$ ) hold.

\section{Existence and uniqueness of the solution of the moduli problem}

We treat only the special case where all the ringdomains are punctured disks. 
The or e m 5. Let $\left\{\gamma_{j}\right\}_{j=1, \ldots, p}$ be an admissible system of Jordan curves on a hyperbolic Riemann surface $R$, with maximal moduli $M\left(\gamma_{j}\right)=\infty$ for all $j$. Let $m_{j}$ be arbitrary real numbers, normalized such that $\sum_{j} m_{j}=0$. Then there is a holomorphic quadratic differential $\varphi$ on $R$ with closed trajectories, of homotopy type $\left\{\gamma_{j}\right\}$, and such that the reduced moduli $M_{j}$ of its punctured disks satisfy the equalities

$$
M_{j}=m_{j}+c
$$

for some $c$ independent of $j . \varphi$ is uniquely determined up to a positive constant factor.

To prove uniqueness, let $\varphi$ and $\tilde{\varphi}$ be two solutions, with $M_{j}=m_{j}+c$, $\tilde{M}_{j}=m_{j}+\tilde{c}$ for all $j$. As the system $R_{j}$ belongs to a quadratic differential, it satisfies (II) when compared with the system $\tilde{R}_{j}$ of $\tilde{\varphi}$ :

$$
\underset{j}{\operatorname{Min}}\left\{\tilde{M}_{j}-M_{j}\right\}=\tilde{c}-c \leq 0 \text {. }
$$

Similarly, starting with $\tilde{\varphi}$, we get $c-\tilde{c} \leq 0$. Therefore $\tilde{c}=c$, hence $\tilde{R}_{j}=R_{j}$ for all $j$. The consequence $\tilde{\varphi}=$ const $\cdot \varphi$ is immediate.

The existence is easily established by means of the previous existence theorem. We first notice that there are disjoint punctured disks $R_{j}^{\prime}$ with reduced moduli $M_{j}^{\prime}=m_{j}+c^{\prime}$ for some $c^{\prime}$. We now make $c^{\prime}$ as large as we can. By a normal family argument there exists a maximal system $R_{j}$. We choose the conformal mappings of the $R_{j}$ onto punctured disks $0<$ $\left|\zeta_{j}\right|<\varrho_{j}$, with $\left(d \zeta_{j} \mid d z_{j}\right)(0)=1, z_{j}$ the given parameters near the points $Q_{j}$, as local homeomorphisms. Let $0<\varrho<\varrho_{j}$ for all $j$ and denote by $R_{j}(\varrho)$ the ringdomains corresponding to the annuli $\varrho<\left|\zeta_{j}\right|<\varrho_{j}$ on $R$, with moduli $M_{j}(\varrho)$. This system is extremal, on the truncated surface $R(\varrho)$, in the direction $\left(M_{1}(\varrho), M_{2}(\varrho), \ldots, M_{p}(\varrho)\right)$, in the sense of the earlier existence theorem. For otherwise we would have a system $M_{j}^{\prime}(\varrho)$ with $M_{j}^{\prime}(\varrho)=(1+\varepsilon) M_{j}(\varrho)$ for some $\varepsilon>0$. Adding the disks $\left|\zeta_{j}\right| \leq \varrho$ to the ringdomains $R_{j}^{\prime}(\varrho)$ we would get a system of punctured disks $R_{j}^{\prime}$ with

$$
M_{j}^{\prime} \geq M_{j}^{\prime}(\varrho)+\frac{1}{2 \pi} \log \varrho>M_{j}(\varrho)+\frac{1}{2 \pi} \log \varrho=M_{j} .
$$

Therefore the system $R_{j}(\varrho)$ is associated with a quadratic differential $\varphi_{\varrho}$. Its trajectories are the circles $\left|\zeta_{j}\right|=$ const which are independent of $\varrho$. But then the $\varphi_{e}$ are just the restrictions of a quadratic differential $\varphi$ on $R$ with the $R_{j}$ as characteristic punctured disks.

As before, we can now introduce the surfaces of moduli. The following facts can easily be established along the same lines as in the earlier proof. 


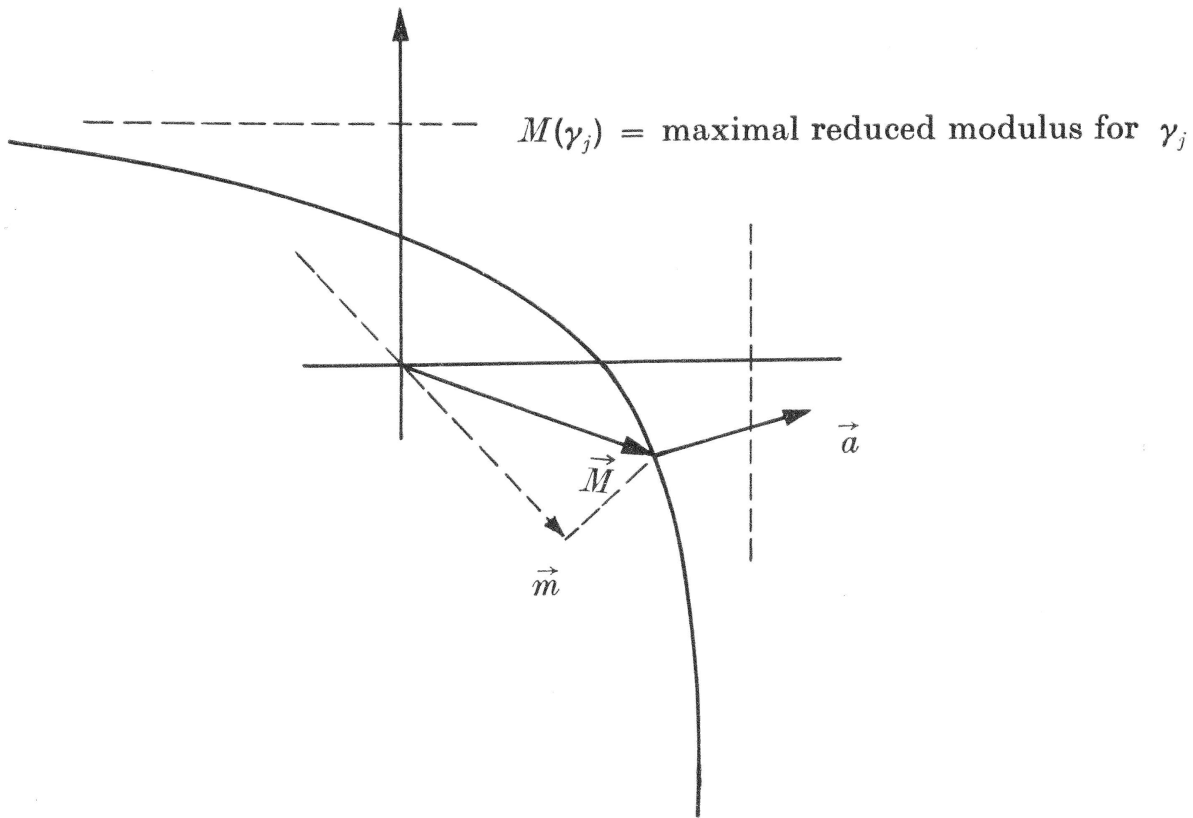

a) Continuity. The vector of (reduced) moduli $\vec{M}=\left(M_{1}, \ldots, M_{p}\right)$ is a continuous function of the vector $\vec{m}$ of parameters $m_{j}, \sum m_{j}=0$. In fact, for $\vec{m} \rightarrow \vec{m}_{0}\left(m_{j} \rightarrow m_{j 0}\right)$ the quadratic differentials $\varphi$ converge to $\varphi_{0}$, if properly normalized, in the sense that $\left\|\varphi-\varphi_{0}\right\| \rightarrow 0$.

b) Convexity. Tangent plane. The plane

$$
\sum_{j} a_{j}^{2}\left(X_{j}-M_{j}\right)=(\vec{a}, \vec{X}-\vec{M})=0,
$$

with $\vec{a}=\left(a_{1}^{2}, \ldots, a_{p}^{2}\right)$, is a plane of support, with only the point $\vec{M}$ itself in common with the surface of moduli. It is in fact the tangent plane at this point, and the normal vector $\vec{a}$ varies continuously with $\vec{m}$.

c) Extremal length problem. The range of directions of the normal vector $\vec{a}$ is the full open quadrant, i.e. $\vec{a} /|\vec{a}|$ is an arbitrary positive unit vector. We can place a tangent plane with this normal at some well determined point $\vec{M}$ of the surface of moduli and then multiply the quadratic differential by an appropriate factor such that the lengths of its trajectories are the given numbers $a_{j}$. The resulting quadratic differential is the solution of the extremal length problem of minimizing the reduced norm with lengths $\geq a_{j}$ in the classes $\gamma_{j}\left(|\varphi(z)|^{1 / 2}|d z|\right.$ is the extremal metric). As the leading coefficients of $\varphi$ at the $Q_{j}$ in terms of any parameter $z_{j}$ are $-A_{j}=$ $-\left(a_{j} / 2 \pi\right)^{2}$, this shows that these coefficients can be arbitrarily given negative numbers. Then there is exactly one quadratic differential $\varphi$ with closed trajectories the characteristic ringdomains of which are the $R_{j}$ and for which $a_{j}=\int_{\alpha_{j}}|\varphi(z)|^{1 / 2}|d z|$. 


\section{References}

[1] STREBEL, KURT: Über quadratische Differentiale mit geschlossenen Trajektorien und extremale quasikonforme Abbildungen. - Festband zum 70. Geburtstag von Rolf Nevanlinna, Springer-Verlag, Berlin-HeidelbergNew York, 1966, 105-127.

[2] - - Bemerkungen über quadratische Differentiale mit geschlossenen Trajektorien. - Ann. Acad. Sci. Fenn. Ser. A I 405, 1967, 1-12.

[3] - - On quadratic differentials with closed trajectories and second order poles. - J. Analyse Math. 19, 1967, 373-382.

Universität Zürich

Mathematisches Institut

CH-8032 Zürich

Switzerland

Received 5 September 1975 